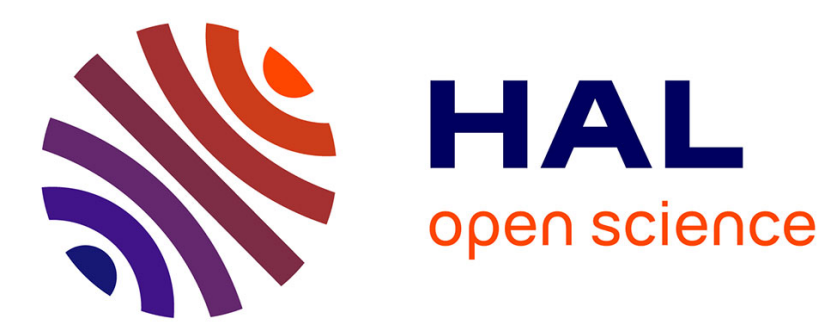

\title{
Interdépendances marchandes et solidarité par la monnaie dans le trueque argentin
}

\author{
Hadrien Saiag
}

\section{To cite this version:}

Hadrien Saiag. Interdépendances marchandes et solidarité par la monnaie dans le trueque argentin. Finance et bien commun, 2010, 37-38 (2), pp.104. 10.3917/fbc.037.0104 . halshs-02343374

\section{HAL Id: halshs-02343374 \\ https://shs.hal.science/halshs-02343374}

Submitted on 2 Nov 2019

HAL is a multi-disciplinary open access archive for the deposit and dissemination of scientific research documents, whether they are published or not. The documents may come from teaching and research institutions in France or abroad, or from public or private research centers.
L'archive ouverte pluridisciplinaire HAL, est destinée au dépôt et à la diffusion de documents scientifiques de niveau recherche, publiés ou non, émanant des établissements d'enseignement et de recherche français ou étrangers, des laboratoires publics ou privés. 


\title{
INTERDÉPENDANCES MARCHANDES ET SOLIDARITÉ PAR LA MONNAIE DANS LE TRUEQUE ARGENTIN
}

\author{
Hadrien Saiag
}

De Boeck Université | Finance \& Bien Commun

\author{
2010/2 - No 37-38 \\ pages 104 à 124
}

ISSN 1422-4658

Article disponible en ligne à l'adresse:

http://www.cairn.info/revue-finance-et-bien-commun-2010-2-page-104.htm

Pour citer cet article

Saiag Hadrien , «Interdépendances marchandes et solidarité par la monnaie dans le trueque argentin », Finance \& Bien Commun, 2010/2 No 37-38, p. 104-124. DOI : 10.3917/fbc.037.0104

Distribution électronique Cairn.info pour De Boeck Université.

(c) De Boeck Université. Tous droits réservés pour tous pays.

La reproduction ou représentation de cet article, notamment par photocopie, n'est autorisée que dans les limites des conditions générales d'utilisation du site ou, le cas échéant, des conditions générales de la licence souscrite par votre établissement. Toute autre reproduction ou représentation, en tout ou partie, sous quelque forme et de quelque manière que ce soit, est interdite sauf accord préalable et écrit de l'éditeur, en dehors des cas prévus par la législation en vigueur en France. II est précisé que son stockage dans une base de données est également interdit. 


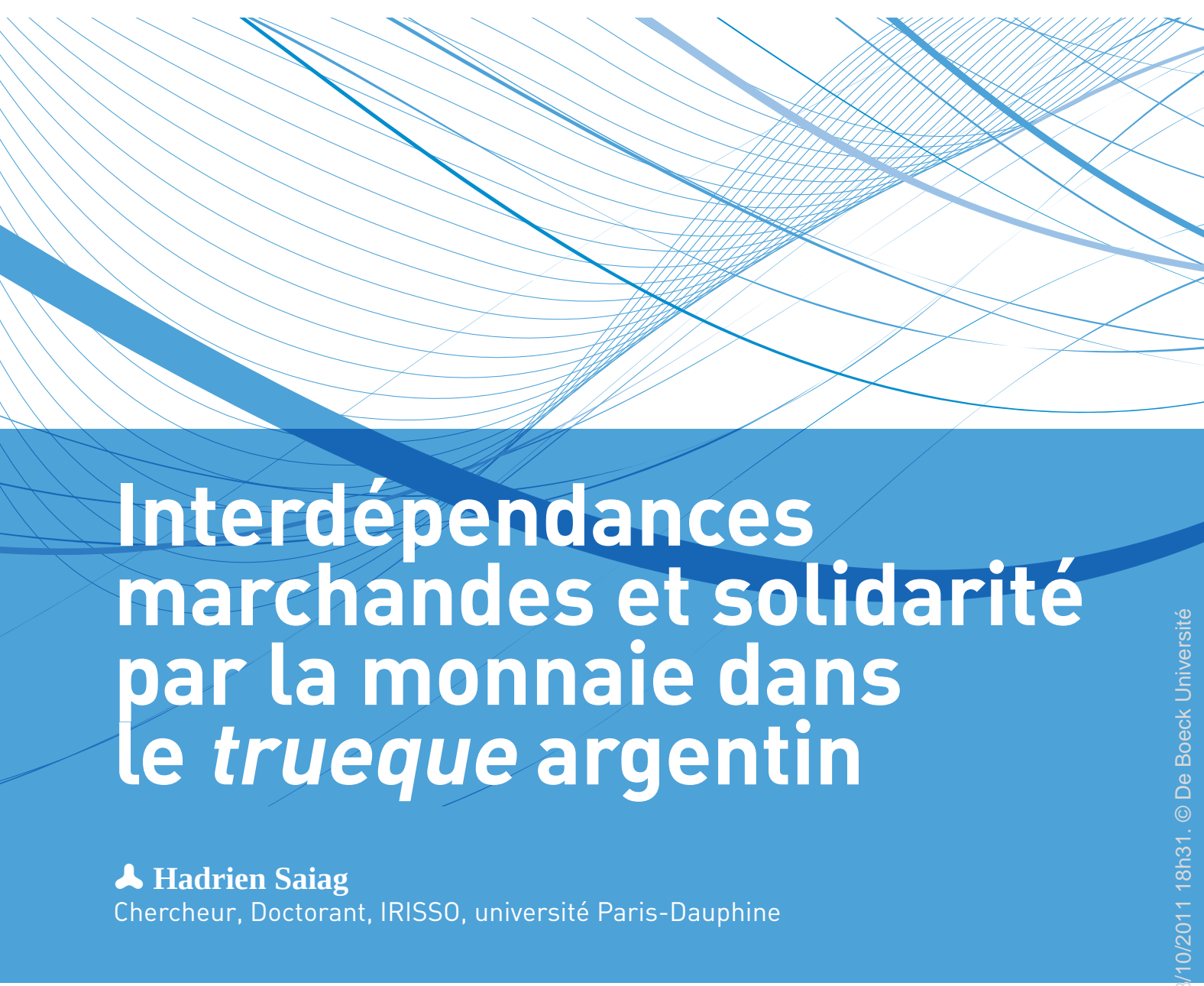

FR Ainsi, les expériences de trueque à l'œuvre dans deux localités voisines, Rosario et Capitán Bermúdez (Poriajhú) sont fort contrastées. L'étude des modalités d'accès aux moyens de paiement (libellés en crédito) est au cœur de notre argumentation : nous proposons d'en déduire la forme que prennent les interdépendances et la relation au groupe. Or, elles diffèrent grandement entre Rosario et Poriajhú : dans les premiers, certains groupes sociaux se trouvent dans une situation dominatrice, car ils sont à même de passer fréquemment d'un système de compte à l'autre (crédito/ pesol. Au contraire, le maintien d'une parité entre le compte en peso et en crédito au sein de Poriajhú ne permet pas ce passage : l'accès aux moyens de paiement met ses participants sur un pied d'égalité. Une question majeure reste cependant en suspend : comment penser un régime de monnayage à même de soutenir l'activité économique tout en mettant ses utilisateurs sur un pied d'égalité ? 
EN The experiences of trueque in two neighbouring towns, Rosario and Capitán Bermúdez (Poriajhú) are very contrasted. The study of the methods of access to the means of payment (made out in crédito) is the object of our argumentation : the idea is to relate access to payment to the forms interdependencies assume within each group. Now, they differ a lot in Rosario and in Poriajhú. In the first, some social groups are in a dominant situation, because they can frequently switch from one accounting system to another (crédito/peso). Maintaining a parity between account in peso and in crédito in Poriajhú does not allow this passage from one to another: the access to the means of payment puts all participants on an equal footing. A fundamental question remains : how to conceive a currency system capable to sustain the economic activity, putting at the same time its users on an equal footing?

\section{Introduction}

Le pluralisme monétaire est récurent en Argentine: outre la monnaie nationale (le peso), des monnaies fiscales émises par les provinces (Théret et Zanabria, 2009) ont circulé, à côté de ce qu’il est convenu de nommer trueque (voir Luzzi, 2005 ; Gómez, 2008 ; Plasencia et Orzi, eds., 2007). Ce terme désigne un ensemble de systèmes monétaires dits " complémentaires » (Blanc 2006). Il fut créé en 1995 dans une banlieue de Buenos Aires à partir de l'idée de trois militants écologistes. Au début de l'expérience, une vingtaine de personnes se réunissaient chaque semaine pour échanger leurs excédents dans le garage de l'un des fondateurs, sur la base d'un système de compte propre. Très vite, les médias se sont intéressés à l'expérience et elle s'étendit à travers le pays. Des moyens de paiement sous forme de monnaie manuelle (billets généralement libellés en " créditos ») valable dans l'ensemble des foires - ferias - qui constituaient alors le trueque remplacèrent le système de compensation pure en vigueur. De là est née une structuration en réseaux nationaux des diverses expériences. Depuis, ces réseaux ont pour la plupart disparu et le trueque s'est reconstitué sur des bases plus locales.

\section{Deux expériences de Trueque}

Ainsi, les expériences de trueque à l'œuvre dans deux localités voisines, Rosario et Capitán Bermúdez (petite ville située dans sa banlieue) sont fort contrastées. C'est sur elles que ce texte se base. (Les données présentées ici ont été obtenues grâce à un travail de terrain mené entre août et décembre 2009 dans ces deux localités. Nous nous sommes basés sur des observations de pratiques monétaires (trueque) et sur dix-sept entretiens semidirectifs consacrés à l'étude des budgets familiaux). À Rosario, il subsiste, à notre connaissance, trois lieux de ferias (foires) : un terrain vague («El campito » - trois fois par semaine), la place

Trueque: this term refers to a series of "complementary" monetary systems (Blanc 2006). The term was coined in 1995 in a suburb of Buenos Aires, elaborating on an idea aired by three environmental activists.

The then used notional compensation system was replaced by a new physical means of payment, called "créditos" notes, valid throughout the fairs or ferias constitutive of the trueque.

Thus, the trueque experiments made in the two neighboring towns of Rosario and Capitán Bermúdez (Poriajhú) are very different. 
As Jean-Michel Servet (2010) understands it, the term solidarity refers to situations where reprocity takes precedence over market and redistribution principles (see also Servet 2007 and Hillekamp, this issue).

\footnotetext{
Within the integration principle, everybody is defined both through his being part of society as a whole and the mutual web of relationships he entertains with others. From this, follows the original meaning of the reciprocity principle, as it registers within the context of a social whole meant to be such, while acknowledging everybody's specificity on an equality basis. (termed symetrics by Polanyi).
}

Alem (deux fois par semaine), et une association de quartier (deux fois par semaine) - les noms des lieux et des enquêtés ont été modifiés afin de préserver l'anonymat. Chacune de ces ferias regroupe entre quinze et 100 personnes. Les moyens de paiement, sous forme de monnaie manuelle, y sont émis par ceux qui assuraient déjà leur émission à l'époque où le trueque à Rosario intégrait l'un des réseaux nationaux. La faible institutionnalisation du trueque à Rosario contraste avec celle de Capitán Bermúdez. Dans cette petite ville, le trueque s’intègre dans les activités plus larges d'une organisation luttant pour la libération des plus pauvres: Poriajhú (Poriajhú signifie « les pauvres » en guarani). Les trois ferias de la ville (une par semaine) regroupent chacune entre dix et trente personnes, dont une majorité bénéficie également de microcrédits (en peso) octroyés par Poriajhú.

\section{Solidarité, réciprocité et intégration}

Ces expériences sont analysées dans la lignée des travaux de Karl Polanyi (voir en particulier Polanyi, 1983/1944 et Polanyi, Arensberg et Pearson, 1957). Par solidarité, il faut entendre avec Jean-Michel Servet (2010) les configurations dans lesquelles le principe de réciprocité domine ceux du marché et de la redistribution (voir également Servet, 2007 et Hillenkamp, ce numéro). Une telle définition fait sens si l'on abandonne l'approche néoinstitutionelle qui associe les principes d'intégration à de simples modalités de transfert, ce qui, implicitement, « part des individus pour comprendre le tout social » (Servet 2007, 263). L'articulation entre les principes d'intégration est à la base de l'« organisation des sociétés » (Servet 2010), et pas uniquement des modalités de transfert. Cela implique de les saisir au niveau des « croyances [...] que les sociétés occidentales auraient intégrées au point d'en faire des dogmes » (Servet 2007, 263). Les principes d'intégration définissent donc à la fois l'inscription de chacun dans la totalité sociale et la forme des relations qu'ils nouent les uns avec les autres. C'est sur ce point que le principe de réciprocité tire son originalité : à travers lui, il y a inscription dans un tout social pensé comme tel (cette inscription est revendiquée en tant que telle) et une reconnaissance des singularités de chacun sur une base égalitaire (la symétrie chez Polanyi). Le marché se situe aux antipodes : il nie la totalité sociale (les interdépendances y sont mécaniques, résultantes des actions individuelles) et met les identités entre parenthèses, faisant comme si nous avions à faire à des individus interchangeables. Enfin, la réciprocité se distingue de la redistribution car en l'absence de structure pyramidale, il ne saurait être question de soumission à un rapport hiérarchique. 
La thèse développée ici est la suivante : l'étude de la forme prise par la monnaie permet, dans le cas du trueque, de déterminer l'articulation entre les modes d'institution de l'économie qui le constituent (lequel domine ?). Parce qu'elle se situe au niveau du pouvoir, la monnaie détermine s’il y a égalité fictive, dissimulant des dominations (place de marché) ou égalité de fait entre les composantes d’un collectif (réciprocité). En outre, en participant à la reproduction des groupes, elle pose la question de la relation à la totalité sociale.

La première partie traite de la coexistence des modes d'institution au sein du trueque. Dans une seconde partie, nous montrons que derrière le modèle de place de marché se trouve en fait, à Rosario, une hiérarchie fondée sur les modalités d'accès aux moyens de paiement, alors que celles-ci instaurent des relations entre égaux à Poriajhú. Enfin, la troisième partie tente d'expliquer cette divergence à partir de la place qu'occupe la monnaie dans la reproduction des groupes formés par les participants au trueque en se basant sur la distinction entre autorité et pouvoir, formulée par Louis Dumont.

\section{Le trueque articule différents principes d'intégration}

Les trois principes d'intégration de l'économie dégagés par Karl Polanyi structurent le trueque à divers niveaux. La redistribution apparaît surtout en amont, dans la circulation de biens délivrés par les politiques d'assistance aux pauvres. Les relations se nouant lors des ferias, où biens et moyens de paiement circulent, font quant à elle appel, en proportion variable, au principe de marché et de réciprocité.

\section{Le principe de redistribution}

Le principe de redistribution se trouve généralement en amont des expériences dont il est ici question. À Rosario, cela passe par l'échange de produits provenant des politiques d'assistance aux pauvres : un grand nombre d'entre elles se traduisent par la distribution de sac (bolsón) de nourriture de première nécessité : lait en poudre, boites de conserve, pâtes, riz, etc. Lorsque cette aide s'avère être incohérente avec les besoins des ménages qui en bénéficient, ces derniers proposent certains biens à l'échange (principalement le lait en poudre). À Poriajhú, la redistribution, plus discrète, est présente à travers un programme de microcrédit («banquito popular de la buena fe », $c f$ de Nigris, 2008) : les ferias ont lieu chaque semaine lors du remboursement des prêts octroyés par Poriajhú à partir de fonds provenant du budget de l'État national. Ces fonds facilitent le contact de Poriajhú avec les populations locales à travers l'octroi, la rémunération des
The trueque is structured along the lines of Karl Polanyi's three economic integration principles.

The redistribution principle operates as a rule prior to practical experiments on the ground. In Rosario, it is exemplified by the exchange of products assistance policies have made available to poor people. 
The market principle lies at the heart of the trueque, bringing people together as equasl (the market place), while still entering them into commercial relationships. The market place venue brings puts people in a salesplace situation.

\section{On the market}

place however, the spontaneous character of ferias relationships does not apply, as people interact as clients. personnes encadrant l'octroi de microcrédits (en peso) et, par là, supervisant le trueque. Cependant, la redistribution est rarement au cœur du trueque.

\section{Le principe de marché}

Le principe de marché est en revanche fortement présent au sein du trueque, à travers une articulation entre l'égalité fictive des participants (de la place de marché) et la reproduction de liens les unissant dans le temps (liens de clientèle). Le rapport de type “place de marché” oblige à « jouer à la marchande » : il y a « neutralisation momentanée des identités sociales » (Servet, ed. 1999). Ainsi, des personnes d'horizons divers, qui ne se côtoient pas en en dehors du trueque, sont amenées à y interagir. J'ai pris conscience de ce point à travers les grandes difficultés auxquelles j'ai dû faire face, au début de mon travail de terrain, au moment de proposer un entretien à ses participants : alors que ceux-ci étaient disposés à me raconter plus que ce que je leur demandai lors des la ferias, une fois celles-ci terminées, je me heurtai à un refus gêné. En demandant d'en savoir plus sur leur vie, audelà trueque, je brisai en fait un tabou révélateur de l'emprise du rapport de place de marché : cela conduisait implicitement à montrer que l'égalité n’était que formelle.

Cependant, l'autre face de la place de marché, l’instantanéité des relations, ne se retrouve pas dans les ferias : les liens de clientèles y occupent une place centrale. Lorsque les femmes se regroupent pour aller ensemble au trueque sur Rosario en louant une camionnette (ce que font la majorité des femmes vivant à Capitán Bermúdez), les liens de clientèle sont mobilisés avant que ne débutent les échanges. Elles profitent de cette location collective pour s'approvisionner à bons prix auprès d'un grossiste situé à la périphérie de la ville en biens qu'elles consommeront ou qu'elles proposeront à la feria. Une fois sur place, leur première préoccupation est de régler ou se faire régler les dettes qu'elles ont contractées avec d'autres participantes : par le report des soldes non équilibrés de feria en feria, ces dettes unissent créanciers et débiteurs à travers le temps. Les liens de clientèle sont également mis à profit afin d'obtenir des informations précieuses : heure, date et lieu des différentes ferias (car cela change beaucoup), prix des biens proposés et éventuelles bonnes affaires. Ainsi, lorsque les femmes viennent en groupe, l'une d'entre elles est (presque) toujours à l'affût des nouveaux arrivants ou d'un prix plus bas que les autres : le temps du trueque est extrêmement rapide, et il est possible de vendre l'ensemble des marchandises que l'on propose en moins d'une minute. Enfin, dans l'une des foires que nous avons pu observer, tisser des liens de clientèle est également une condition nécessaire à l'utilisation des créditos : le vendredi à « El campito », la majorité des échanges a lieu en 
peso ; le crédito est réservé à un réseau d'utilisateurs relativement fermé.

Si forts soient-ils, les liens de clientèles ne doivent pas être confondus avec la réciprocité. En effet, comme il a été indiqué en introduction, la réciprocité n’est pas un lien interindividuel, mais un lien au groupe qui prend une forme particulière : celle de la reconnaissance des interactions dans une totalité sociale pensée en tant que telle. Or, ni la totalité sociale, ni les interdépendances entre les participants ne sont saisies à travers les liens de clientèle. Les identités sont bien « mises entre parenthèses » : à Rosario, en dehors des ferias, on ne se connaît plus. Plus encore, lors des ferias, ignorant (presque) tout des autres participants, il est commun de les nommer à partir des biens qu'ils proposent à l'échange ("la verdulera [celle qui vend des fruits et légumes] ", " la que vende jugos [celle qui vend des jus] », etc.). En l'absence de relation au groupe pensé en tant que tel, les liens de clientèle ne mettent pas à mal l'une des caractéristiques centrales du marché en tant que principe d'intégration de l'économie : les interdépendances y sont involontaires, il s'agit d’une conséquence mécanique des actions individuelles.

\section{Le principe de réciprocité}

Enfin, la réciprocité se trouve tantôt en aval des ferias, tantôt au cœur de celles-ci (selon les cas). En aval, on la retrouve dans toutes les configurations à travers la répartition au sein des foyers des fruits du trueque. Les règles qui régissent l'organisation des budgets familiaux sont extrêmement variées et il ne saurait être question de toutes les exposer ici (voir par exemple Guérin, 2008). Notons simplement qu'il y a toujours, d'une part, reconnaissance d'un tout irréductible aux membres du foyer et, d'autre part, des spécificités de ses membres. Le premier point est perceptible notamment à travers l'expression utilisée par Angela afin de qualifier les dépenses qu'elle effectue pour le compte du ménage : elle « divise » l’argent... qui a donc dû être centralisé afin de servir les fins du foyer en tant que tel. La reconnaissance des individualités prend des formes diverses, selon les modalités de gestion des budgets : elle peut passer par la répartition des produits extraits du trueque entre les membres d'un foyer (la grand-mère ayant droit aux biens permettant de nourrir sa petite fille lorsqu'elle déjeune chez elle ainsi que ceux qu'elle apprécie le plus, et la mère garde le poisson et l'huile, qu'elle utilise ensuite afin confectionner des aliments qu'elle vendra par la suite en peso), ou, plus simplement, par la formation d'un « pot commun » dans lequel chacun peut se servir selon ses nécessités. Enfin, les participants au trueque de Poriajhú font également état (sans la nommer ainsi) de la réciprocité au sein même des foires :
However strong, client relationship should not be confused with reciprocity, which is not an interpersonal relationship but relates individuals to a group assuming thereafter a peculiar form. Here, interactions take place within a social whole recognized as such.

Participants of the Poriajhú trueque evoke, without calling it by this name, reciprocity within the context of fairs. 
[Le trueque] est [...] un moyen d'aider les autres. C'est une manière de s'impliquer dans la nécessité des autres. Car ici il y a des familles dont la situation économique est précaire. Alors, ils se détachent de certains biens, une chemise, un pantalon, etc., pour les échanger contre des biens alimentaires [mercaderia], du sucre, etc. Et tu vois c'est réciproque, parce que de mon côté j'ai besoin d'un pantalon et elle le propose, je lui donne un kg de sucre, de yerba [herbe à la base de la préparation du mate, boisson très populaire en Argentine] et j'ai le pantalon. C'est comme... comment le dire... c'est comme partager les nécessités (Entretien avec Nelina, Capitán Bermúdez, décembre 2009, souligné par moi-même)

Ainsi, on retrouve les trois principes d'intégration de l'économie dans le trueque, mais la question de leur articulation reste entière. Or elle est primordiale, dans la mesure où ces principes sont traversés par des tensions internes (place de marché/liens de clientèle) et entre chacun d'entre eux (réciprocité/marché). Pour que la situation ne soit pas explosive, il est donc nécessaire que l'un des principes domine les autres. C'est cette analyse que permet d'entreprendre l'étude des modalités d'accès aux moyens de paiement au sein du trueque : il convient de comprendre s'il y a ou non égalité effective entre les participants, puis de saisir les rapports au groupe qui sous-tendent ces diverses modalités d’accès.

Reviewing how

people manage to secure créditos as a means of payment lies at the core of our demonstration, since this is instrumental to understanding the way in which they relate to the group. Now, this relationship varies greatly between Rosario and Poriajhú. In the first instance, certain social groups are in a dominating position, for they pass easily from one type of account to the other (crédito/peso). When there is a fixed rate of exchange between the two currencies however, as it is the case in Poriajhú, this passage is not possible, while access to means of payment puts all participants on a equal footing.

\section{Derrière les modalités d'accès à la monnaie, un rapport hiérarchique?}

L'étude des modalités d'accès aux moyens de paiement (libellés en crédito) est au cœur de notre argumentation : nous proposons d'en déduire la forme que prennent les interdépendances et la relation au groupe. Or, elles diffèrent grandement entre Rosario et Poriajhú : dans les premiers, certains groupes sociaux se trouvent dans une situation dominatrice, car ils sont à même de passer fréquemment d'un système de compte à l'autre (crédito/peso). Au contraire, le maintien d'une parité entre le compte en peso et en crédito au sein de Poriajhú ne permet pas ce passage : l'accès aux moyens de paiement met ses participants sur un pied d'égalité.

\section{Monnaie, compte, paiement et dette}

Il est nécessaire de préciser le sens de certains concepts avant d'aller plus en avant dans l'argumentation. L'approche interdisciplinaire du fait monétaire, telle qu'elle a émergé à partir des recherches menés autour de Michel Aglietta et André Orléan (eds., 1998), puis de Bruno Théret (ed.,2007), est considérées comme acquise : la monnaie est un système de compte et de paiement des dettes. Elle se trouve au cœur de la reproduction des sociétés car elle n’est pas définie par rapport à un réel qui lui serait extérieur et premier, mais par ses «propriétés géné- 
riques ». La monnaie n’est pas ce qu'elle fait (Théret 2008b). Elle est en elle-même un rapport social (Ingham 2004). Le compte est ce qui permet le rapport au groupe, car il permet l'évaluation sociale des dettes et créances. Le paiement, quant à lui, donne une unité dynamique au tissu de dettes et créances. Il leur permet de circuler, par un flux ininterrompu de paiement et d'endettement. Détenir un moyen de paiement, c'est détenir une créance sur la communauté de paiement. Enfin, il convient de noter que la monnaie ainsi définie est susceptible de mettre en mouvement des dettes de diverses natures (Théret, 2009) : dettes entre agents privés dont il est possible de se libérer, mais qui inscrivent néanmoins dans une division sociale du travail qui leur préexiste, dettes qui assurent la reproduction des collectifs « en surplomb ", à travers des paiements à des entités suprahumaines (les dieux, l'État à travers l'impôt, etc.) et, enfin dette entre humains ou groupes humains inscrits dans une totalité sociale pensée comme telle. On retrouve donc derrière la pluralité des dettes la pluralité des modes d'institution polanyiens.

\section{Régimes de monnayage}

Les conditions d'accès aux moyens de paiement sont abordées à travers l'insertion des régimes de monnayage dans les rapports sociaux de production. Un régime de monnayage désigne l'ensemble des modalités de création et de destruction des moyens de paiement dans le temps (Théret 2008b). Ainsi, il est par exemple possible de distinguer deux régimes de monnayage contrastés: celui des monnaies fiscales (Théret et Zanabria 2009) et celui des monnaies bancaires privées. Dans le premier cas, créations et destructions monétaires répondent aux besoins de financement de l'État, alors que les besoins des agents privés en sont les déterminants principaux dans le second. Cependant, régime de monnayage et condition d'accès aux moyens de paiement ne sont pas synonymes : certains groupes sociaux n'ont un accès privilégié aux moyens de paiement seulement car d'autres en sont exclus. C'est en ce sens qu'il est convient d'étudier l'interaction entre les régimes de monnayage et les rapports sociaux de production dans lesquels ils s’insèrent.

\section{Les modalités d'accès aux moyens de paiement}

Dans le trueque, les modalités d'accès aux moyens de paiement ne se confondent pas avec celles qui viennent d'être décrites. La création des moyens de paiement repose principalement soit sur l'appartenance au groupe (Poriajhú), soit sur l'usage dans des pratiques de paiement de biens qui, en dehors du trueque, ne remplissent pas cet office. Or, tous n’y ont pas accès dans la même mesure, du fait du système de compte. Avant de décrire les différentes modalités d'accès aux moyens de paiement en vigueur dans le trueque, il convient d'insister sur le rôle des usa-
Currency regime and access conditions to means of payment are not synonymous: some social groups have a privileged access while others are excluded. This sheds some light to the interaction between currency regimes and the social production relationships within which they take place. 
At bottom, privileged access to crédito in Rosario is due to certain participants' better integration in the peso's circulation area.
In other words, goods offered by Stefani in créditos are not as highly valued as those sold by Luis in pesos. It is in Stefani's interest to go from one sphere to the other. ges monétaires : il n’y a monnaie que dans des « pratiques socialisées de compte et de paiement » (Blanc 2000) : la forme prise par les moyens de paiement importe peu, pourvu que le paiement s'articule à un système de compte. Ainsi, certains groupes peuvent avoir accès à des moyens de paiement libellés en crédito à travers l'usage dans des pratiques de paiement de biens qui en dehors du trueque ne sont pas des moyens de paiement (savon, purée de tomates, farine, etc.). Nous retrouvons à Rosario une situation proche de celle analysée par Pierre Alary (2006) au Laos : c'est le passage d'un système de compte à l'autre à travers les moyens de paiement qui donne (ou non) à certains groupes une position dominante. C'est ce que nous tentons de montrer à présent, en distinguant trois modalités d'accès selon trois types de participants au trueque à Rosario.

\section{Passage de la sphère du peso à celle du crédito : tirer profit d'une bonne intégration dans la sphère du peso}

À Rosario, la première forme d'accès privilégié au crédito trouve son origine dans la relativement meilleure intégration de certains participants dans la sphère de circulation du peso. Illustrons ce cas à travers Eleonora, Angela (sa fille) et l'une de leurs amies, Stefani. Les budgets de leurs foyers et leurs trajectoires au sein du trueque présentent un certain nombre de points communs. En premier lieu, leur implication dans le trueque n'a pas pour motif initial la nécessité économique. Leur accès au peso est donc relativement bon. Pour Stefani, cela passe essentiellement par les revenus de son mari (il possède une entreprise de transport routier, vend une partie de la pèche qu'il effectue sur le fleuve Paraná, et s'occupe d'un centre de lavage d'automobiles situé dans un quartier résidentiel) et, accessoirement, par sa fille (salariée auprès d'une mutuelle de santé). Elle possède en outre ses propres entrées en peso à travers divers services qu'elle propose aux personnes âgées de son quartier (les emmener chez le médecin, chercher leurs médicaments, leur retraite, etc.). De leur côté, Eleonora et Angela sont directement insérées dans la sphère de circulation du peso à travers des " microemprendimientos » (activités génératrices de revenus non salariées et dont la rentabilité est faible) plutôt originaux et pour lesquels il existe une demande solvable.

Cet accès privilégié au peso leur donne une position dominante au sein du trueque. Pour le comprendre, examinons en détail deux transactions parallèles, l'une engageant Eleonora avec Luis, et l'autre entre ce dernier avec Stefani (tableau 1). Luis travaille dans l'un des marchés de gros de fruits et légumes de la ville de Rosario, et est payé, en partie, en nature. N'ayant que faire des fruits et légumes, il en propose une partie à « El campito », en créditos. Dès son arrivée sur place, il cherche Eleonora et Stefani afin de les leur proposer (liens de clientèles). Dans un 
Tableau 1 : Résumé des échanges Luis/Eleonora et Luis/Stefani (en créditos) :

\begin{tabular}{|lr|lr|lr|r|}
\hline Rafael => Marta & & Eleonora => Rafael & & Luis => Yoli & & Stefani => Raphael \\
\hline Tomates (2Kg) & 5000 & Sel (2 paquets) & 2000 & Courgettes (2) & 1500 & Huile (2l) \\
Courgettes (2) & 2000 & Sucre (3Kg) & 10500 & Pommes (2 Kg) & 5000 & \\
Ail (3 gousses) & 4500 & Savon (1) & 4000 & Mandarines (1Kg) & 2000 & \\
Pomme (1Kg) & 2000 & Total avant compensation & 16500 & Carotte (1Kg) & 1500 & \\
Mandarine (2000) & 2000 & & & Fraises (1Kg) & 2000 & \\
Courge (1 pièce) & 2500 & Sucre (1Kg) & 3500 & Tomate (1Kg) & 2500 & \\
Mandarine (1Kg) & 2000 & & & & & \\
\hline Total & 20000 & Total & 20000 & Total & 14500 & Total \\
\hline
\end{tabular}

Source: enquête de terrain

premier temps, Eleonora choisit ce qui l'intéresse, puis c'est au tour de Luis de faire de même. Luis retourne ensuite là où il est installé le temps de la feria. Pendant ce temps, nos deux protagonistes font leurs comptes : ils évaluent en créditos la valeur des biens qu'ils se sont mutuellement cédés. Puis Luis revient voir Eleonora et ils comparent leurs soldes. Ceux-ci sont (presque) toujours déséquilibrés. Afin de régler les soldes, la débitrice (Eleonora) transmet au créancier (Luis) un bien choisi par ce dernier, dont la valeur évaluée en créditos est égale au montant de sa créance. Une série d'opérations similaires a ensuite lieu entre Stefani et Luis. Avant d'entrer dans le détail des évaluations monétaires des biens mis en circulation, il convient d'insister sur le fait que ceux que Eleonora et Stefani ont transmis à Luis ont été acquis auprès d'un grossiste, en peso. En réglant leurs dettes grâce à ces biens, elles sont donc passées de la sphère du peso à celle du crédito... et cette opération leur est très profitable ! L'extrait de discussion suivant entre Stefani et moi-même à propos de l'opération décrite ci-dessus en témoigne :

Moi : Luis t’a donné deux courgettes [zapallitos]...

Stefani : Courgettes, pommes, tomates, carottes, fraises, mandarines, cela faisait 19.500 créditos. Et je lui ai donné deux bouteilles d'huile, je crois [...]

Stefani : Je lui ai fait chaque bouteille d'huile à 7.500 créditos, mais je les ai payés 3 pesos chacune. C'est mon profit [esto es mi ganancia]

Moi : Et aurais-tu pu lui vendre chaque bouteille à 7,5 pesos [Dans les discussions, la relation implicite peso/crédito à $E$ l campito est de $1 / 1000]$ ?

Stefani : Nonnnnnnnnnn !

Moi : C’est pour cela que le crédito te convient mieux ?

Stefani : Evidemment. Sur la bouteille il est écrit que je l'ai payée trois pesos [...]. Or, Luis ne me donnerait pas tout cela pour 6 pesos ! Regarde tout ce qu'il m’a donné : courgettes, pommes, fraises, carottes, tomates, etc. Tu ne sais pas quelle quantité j’ai obtenue ! (Entretien avec Stefani, St Lorenzo, novembre 2009) 
Other participants do not have a privileged access to peso, but their activities enable them to benefit from the differential between the two monetary spheres. Even though their income is in pesos, the access to this currency depends on créditos activities.
En d'autres termes, le rapport entre les évaluations monétaires des biens proposés par Stefani en créditos et leur évaluation en peso est bien supérieur à celui des biens proposés par Luis : Stefani a donc tout intérêt à passer certains biens d'une sphère à l'autre. Il est difficile de donner des données chiffrées mesurant avec précision cet écart entre évaluations monétaires (crédito/ peso), mais nos observations suggèrent que les produits obtenus à travers le peso sont généralement évalués en créditos de manière plus favorable que les autres (légumes et produits provenant de l'assistance aux pauvres). Le tableau mis en annexe (1) donne plus de précision sur ce point.

\section{Passage crédito/peso : faire l'interface entre les sphères}

Les articulations entre peso et crédito qui viennent d'être décrites ne sont cependant pas les plus courantes : à Rosario, peu participent au trueque car ils ont les moyens de s'en passer. D'autres participants n’ont pas un accès privilégié au peso (en termes quantitatifs), mais leurs activités se prêtent particulièrement à l'articulation des deux sphères monétaires. Même si le revenu de ces foyers prend en partie la forme de pesos, l'étude de leur budget révèle de très fortes interdépendances entre sphères monétaires : leur accès au peso est lié à leur activité en crédito. C’est ce que met en avant le couple formé par Celina et Jorge. Leur accès au peso est relativement limité. Il provient d'abord de la vente du carton récupéré par Jorge parmi les déchets, mais cela représente une somme infime. Deux de leurs trois enfants bénéficient également d'une bourse, qui couvre une partie des frais liés à leur scolarité (achat de cahiers, photocopies, etc.). Enfin, la mère de Celina possède un tout petit commerce où l'on vend de tout (kiosko), dans un quartier plutôt chic de Rosario (près du fleuve Parana). Après s'être fortement endettée il y a quelques années, la mère de Celina s'est trouvée dans l'incapacité d'avancer les sommes nécessaires (en peso) afin d'approvisionner son commerce ; elle a donc demandé à Jorge de l'aider. Entre eux, les recettes sont réparties selon les produits vendus : le produit de la vente des biens introduits par Jorge lui revient, et inversement. L'activité de Jorge dans le kiosko permet donc au foyer d'accéder dans une plus grande mesure au peso, mais cette activité est fortement liée à l'action du couple dans les différentes ferias de trueque de Rosario. Outre des vêtements usagés et neufs (qui correspondent aux invendus de l'une des sœurs de Celina, qui possède une petite « boutique » de vêtements), ils proposent des sprays à gaz aérosol (déodorants, anti-moustique, etc.) et des parfums, qu’ils se sont procurés entre 2005 et 2008 dans les déchets du dépôt d'une entreprise les produisant. Celina s'occupe de la vente des vêtements et, en contrepartie, recherche des aliments de toutes sortes (yaourts, boites de conserve, pâtes, riz, fruits, légumes, sauces diverses, etc.). Il s'agit d'une source non négligeable pour le foyer : elle estime à 70 pesos la valeur des biens ainsi obtenus 
à chaque feria, soit 560 pesos par mois. Son mari, de son côté, « transforme » les gaz aérosol et parfums en nourriture. C'est ici que trueque et kiosko, crédito et peso, s’articulent à partir d'allées et venues parfois complexes entre systèmes de comptes. Illustrons ce point par un exemple. Jorge achète trois savons en promotion dans un supermarché pour 1,40 peso l'unité, puis les revend au trueque 3.500 créditos chacun. Avec 3.000 des 3.500 créditos procurés par la vente de l'un de ces savons, il achète trois éponges (1000 créditos chacune) qu'il revend 2 pesos dans le kiosko. Ainsi, il explique que « de 1,40 peso [... il est] passé à 6 . Et il [... lui] reste en plus 500 créditos ! ». Avec le produit de la vente des éponges, il achète pour 3 pesos un aérosol dont il manque une pièce. Grâce à la réserve de pièces d'aérosol qu'il a accumulée, il le répare et le vend 15 pesos au kiosko.

\section{Passages crédito/peso : articulations réduites}

Enfin, ceux qui ne peuvent pas passer aisément d'une sphère à l'autre constituent un troisième groupe. Le trueque n'occupe pour eux qu'une place marginale car, n’ayant pas d'accès privilégié aux moyens de paiement libellés en créditos, ils se trouvent en position de dominés. Ils survivent généralement grâce aux politiques d'assistance aux pauvres et à des activités précaires, rémunérées en peso et peu articulées avec le trueque. La situation d'Andrea est sur ce point représentative. Elle vit avec son compagnon, Estéban, et leur enfant dans un quartier relativement pauvre de Rosario. À la feria de trueque du quartier St Cristobal, Andrea propose uniquement des vêtements usagés. Il s’agit soit de vêtements qui ne sont plus utilisés par le ménage, soit de donations de proches. Grâce aux créditos procurés par leur vente, Andrea acquiert principalement d'autres vêtements usagés et quelques produits domestiques usagés (rideau, produit anti-moustique, râpe à fromage, etc.). Vu le faible prix relatif auquel sont vendus les vêtements usagés (voir annexe 1), elle ne peut accéder qu'irrégulièrement et en faible quantité aux aliments (pommes de terre, pommes, huile, farine, lait, pâtes, etc.). Par ailleurs, l'accès du couple au peso est également limité. Estéban propose ses services à la journée pour des travaux divers (changitas - peinture, réparations en tout genre), mais cela ne suffit pas à remplir ses jours. De son côté, Andrea nettoie chaque samedi durant deux heures les toilettes et la cuisine d'une entreprise voisine. Le couple obtient la part la plus importante des pesos du ménage lorsqu’ils lavent les voitures qui se présentent devant chez eux (ils ont aménagé pour cela leur « jardin »). Mais là encore, le revenu généré par cette activité est très irrégulier et relativement faible (le couple déclare laver 4 à 5 voitures le samedi, une voiture par jour en semaine. À 15 pesos par lavage, cela représente 510 pesos par mois. A ces revenus très irréguliers s'ajoute, chaque semaine, le lavage d'un camion pour 150 pesos). Ils « satisfont » donc à la plus grande partie des besoins
Those who are not in position to shift from one sphere to the other represent a third group. The trueque is only of marginal interest to them. Without any privileged access to créditos as means of payment, they find themselves in an inferior position. 


\section{Social fragmentation within trueque depend- ing on different access conditions to means of payment highlights the specific case of Poriajhú. Here, interplay between crédito and peso is very limited, while everybody has access to payment means in crédito. In the course of our research on the ground, we have not been told of any reseling in peso of crédito purchased goods.}

alimentaires du foyer en articulant plusieurs politiques d'assistance aux pauvres : celles transitant par l'association des voisins du quartier (vecinal - lait en poudre), par la municipalité de Rosario (le bolson - huile, yerba mate, sucre, sel, pâtes, riz, etc.), et par l'Église (conserves de thon, de viande, sucre, yerba mate). Les ressources tirées de l'assistance aux pauvres sont d'autant plus importantes que ce sont les seules à être régulières. Cependant, elles ne suffisent pas, à elles seules, à maintenir à flot le ménage, qui est poussé à s'endetter chroniquement afin de faire face à l’irrégularité des revenus en peso et en crédito.

\section{Crédito/peso : les différences entre Rosario et Poriajhú}

La fragmentation sociale opérée au sein du trueque sur la base des conditions différenciées d'accès aux moyens de paiement fait apparaître la singularité de la situation à Poriajhú. Ici, les articulations entre crédito et peso sont extrêmement limitées et tous ont accès aux moyens de paiement libellés en crédito (sous forme de monnaie manuelle - billets) avant de participer pour la première fois au trueque. Durant notre enquête de terrain, nous n'avons eu écho d'aucune activité de revente en peso des biens achetés acquis en crédito. Certes, certains produits sont parfois proposés dans le trueque car ils n’ont pas trouvé preneurs dans la sphère du peso, mais cela reste à une échelle très limitée. Les biens proposés proviennent essentiellement des activités productives soutenues par des microcrédits octroyés par Poriajhú. Ainsi, malgré la grande diversité des participants au trueque (y participent un commerçant et fabriquant de vêtements relativement important, des femmes proposant leurs services à domicile bien rémunéré en peso, de petits « microemprendimientos » vivotant grâce au microcrédit et, enfin, des populations plus pauvres ne bénéficiant pas de crédit), il n’est pas question d'une stratification sociale au sein du trueque comparable à celle qui est observée à Rosario. Au contraire, tous sont mis sur pied d'égalité car les conditions d'accès aux moyens de paiement ne dépendent pas de la capacité de groupes sociaux particuliers à articuler peso et crédito. Cela est dû au maintien au sein de ces ferias de la stricte parité entre le peso et le crédito (1/1 pour tous les biens) : dès lors, l'intérêt d’articuler différentes sphères monétaires disparaît.

\footnotetext{
The argument runs as follows: the various relationships between accounting systems as observed on the ground in Rosario and Poriajhú (crédito/peso) are at least in part related to crédito's position in reproducing the fabric of groups.
}

\section{La monnaie, entre pouvoir et autorité}

La thèse qui est défendue ici est que l'existence de relations différentes entre systèmes de compte observés à Rosario et Poriajhú (crédito/peso) est (pour le moins en partie) liée à la position différente qu’occupe le crédito dans la reproduction des groupes : dans le premier cas, il se situe au niveau du pouvoir alors que dans le second il est également présent au niveau de l'autorité. En se référant à Louis Dumont (1983), et à travers Daniel 
de Coppet (1998), La monnaie souveraine distingue l'autorité, comme valeur constitutive du tout social au-delà de ses parties, du pouvoir, en tant que rapport de domination :

L'autorité est un ensemble de valeurs collectives autour desquelles est affirmée la cohésion d'une société. Ces valeurs sont la source de normes qui ordonnent les conduites individuelles. On dira que l'autorité subordonne en valeur le pouvoir. Celui-ci est un rapport de domination fondé sur la possession de moyens permettant à certains individus de dicter leurs conduites à d'autres individus. (Aglietta et al. 1998, 11)

Il convient de préciser que la question est, au-delà de la constitution d'une totalité, celle de la représentation de la totalité en tant que telle. En effet, on pourrait rétorquer à cette présentation succincte que la dimension holiste de la monnaie est indépassable (Orléan 2002) : en passant d'évaluations privées de la richesse à une commune expression de celle-ci, il y a bien objectivation de la valeur à travers le compte et, par là, constitution d'un tout (la société des individus marchands). Ainsi, les dettes privées sontelles pensées comme des relations interindividuelles alors qu'elles inscrivent débiteurs et créanciers dans une division sociale du travail qui les dépasse (Aglietta 2007). Il y a donc bien totalité, mais elle n'est pas pensée en tant que telle. Ainsi, défendre que le crédito ne se situe pas au niveau de l'autorité à Rosario, signifie qu'il ne participe pas à la construction consciente d'une totalité sociale.

\section{Rosario et Poriajhú : un autre sens du crédito}

La section précédente s’est attelée à montrer qu’à Rosario le crédito se situe bien au niveau du pouvoir : c'est à travers un accès privilégié aux moyens de paiement que ceux qui font l'interface entre crédito et peso dominent ceux qui en sont incapables. Le crédito ne se situe pas au niveau de l'autorité car il semble qu'aucun tout cohérent n'émerge à partir du trueque dans cette ville. L'incapacité de la part des émetteurs de la monnaie manuelle libellée en crédito de contrôler l'inflation que connaît le trueque doit d'abord nous alerter sur le manque de confiance éthique (Aglietta et al, 1998) en la monnaie : ses émetteurs n’arrivent pas à mobiliser une représentation commune de l'unité du groupe à même de faire accepter les règles du jeu monétaire.

Au contraire, le projet de Poriajhú va de pair avec la construction d'un tout revendiqué comme tel, que le crédito tend à reproduire. Affirmer la construction d'un tout cohérent peut surprendre, dans la mesure où la grande diversité des activités de Poriajhú peut faire penser que chacune d'entre elles se réfère à un objectif spécifique : la radio, la murga (groupe de musique et de danse) et le travail de récupération des usages d'une langue indigène dans un
As shown in the precedent section, the crédito is very much located at the level of power in Rosario: those who are in a position to take advantage from the trade off between crédito and peso are in a dominant position. Crédito however is not consistent with authority as no overall picture arises out of trueque in this town.

The Poriajhú project, by contrast, goes together with the build up of a cohesive fabric which the crédito tends to reproduce. 
Activities centered around trueque in Poriajhú are meant to build a group on a reciprocal basis. Here, trust looks very much like an implicit reference, for without it the collective set up it aims at promoting would be meaningless. The crédito payment means graphics also points to this aim. bidonville de Rosario peuplé par des Amérindiens Tobas seraient liés à l'appropriation collective de l'espace public, les ateliers d'informatique, de cuisine, de couture, d'artisanat, etc. à l'accroissement des capacités des populations « défavorisées ", le soutien scolaire et l'alphabétisation des adultes à la scolarisation, le trueque, les microcrédits et le local visant à commercialiser les produits élaborés grâce à ces prêts à accroître les revenus des plus pauvres, etc. Or, on ne retrouve pas une telle vision fragmentée dans le projet de celle qui à Poriajhú détiennent l'autorité, Marita (il s’agit du personnage clé de Poriajhú). Elle a gagné une grande reconnaissance parmi les plus démunis du fait de l'action militante qu'elle mène sur le terrain depuis le début des années 1980:

Marita : L'objectif de Poriajhú, sa mission, est de créer des espaces de participations du peuple afin d'affirmer son rôle de protagoniste dans la transformation de la réalité depuis l'éducation populaire [...]. Toutes les activités que nous faisons [...] doivent passer par le contrôle de qualité de cette mission. C'est-à-dire, sommes-nous en train de créer des espaces de participation du peuple afin d'affirmer son rôle de protagoniste dans la transformation de la réalité depuis l'éducation populaire ? Non ? Alors que faut-il changer dans notre pratique pour que cela soit le cas ? Mais tous les travaux de Poriajhú s'inscrivent dans ce cadre. [...] Ce que Poriajhú prétend est donc de réussir à laisser une empreinte à travers ses divers travaux dans les quartiers [...] qui permette de mener à bien implicitement sa mission. (entretien avec Marita, décembre 2009)

Les activités qui entourent le trueque à Poriajhú visent à construire un groupe sur la base de la réciprocité. L'espace manque pour souligner ce point, mais il est utile de mentionner l'une des activités organisées en marge du trueque. Avant de commencer le trueque, les personnes présentes écoutent puis lisent les paroles de la chanson « Maria Maria » (composé par Milton Nascimento). Elle transmet à travers Maria l'image d'une mère universelle dans laquelle tous peuvent se reconnaître. Il s'agit en quelque sorte d'un appel à la révolte, car cette femme se bat au quotidien malgré une existence douloureuse. Après avoir discuté collectivement du texte dans son ensemble, le groupe se divise : chacun est chargé de commenter une partie du texte à partir de son expérience personnelle. De cette manière, tous se l'approprient, mais surtout, les trajectoires personnelles sont exposées et discutées. Il y a donc reconnaissance des individualités au sein d'un groupe considéré comme un tout.

À Poriajhú, le crédito est construit à l'image du groupe et, par là, participe à sa reproduction sur des bases réciprocitaires. Cela passe d'abord par un processus proche de ce que Michel Aglietta (1988) nomme « substitut symbolique » : la monnaie se pare des 
attributs qui donnent au groupe sa cohésion, même si celle-ci prend son essence en dehors d'elle. Cela passe par un processus de symbolisation à travers trois canaux. Il s'agit d'abord du nom de l’unité de compte. « Crédito » signifie « crédit » et, comme en français, son sens est double : crédit/prêt et crédit/confiance (Blanc 1997). Or, la dimension confiance du crédit fait directement référence à la construction d'une totalité, dans la mesure où en matière monétaire il s'agit d'une confiance vis-à-vis du groupe en son ensemble, et non simplement des coéchangistes. La confiance apparaît ici comme une référence implicite à Poriajhú, car sans elle la construction collective qu'il tente de promouvoir n'aurait pas de sens. L'iconographie des moyens de paiements libellés en crédito est également mise à contribution (voir annexe 2). On y retrouve les éléments suivants : «Banco Popular de la Buena Fe », le nom « Poriajhú », son logo ainsi qu'une fourmi. Le premier élément est le nom de l'organisation qui, à l'échelle nationale, est responsable de l'octroi des microcrédits. Il s'agit d'une garantie institutionnelle dont l'unique but est de susciter la confiance : en tant que tel, cet organisme n'est pas lié au trueque. L'inscription du nom de l'organisation « Poriajhù » inclut explicitement le groupe en tant que tel. Son logo est composé d'une marguerite qui prend ses racines en Amérique latine, de briques, du nom de l'organisation, ainsi que de l'inscription « un autre monde est possible ». La marguerite représente l'espoir, ses racines l'unité (utopique) de l'Amérique latine, et les briques évoquent la construction de « l'autre Amérique latine », conforme aux valeurs de solidarité et d'autonomie qui constituent le nodo Poriajhú. La fourmi, quant à elle, représente le travail collectif ainsi que l'union contre les éventuels ennemis. Enfin, le discours de Marita lors de l'instauration de nouveaux moyens de paiement, en 2007, dessine le crédito à l’image de Poriajhú. Elle le compare au peso en commençant par énumérer les avantages de ce dernier : il permet de manger, de s'habiller, de s'éclairer, de téléphoner, etc. Mais il est également, dans son discours, vecteur de vices tels que la vente d'organes, l'accumulation, l'injustice, la pauvreté, le pouvoir, etc. Elle explique alors qu'il incombe au groupe dans son ensemble, par ses usages quotidiens du crédito, de ne pas reproduire les vices du peso (sur la moralité des usages monétaires, voir Parry et Bloch, eds, 1989).

Mais surtout, la monnaie participe à la reproduction du groupe à travers les modalités d'accès aux moyens de paiement. D’une part, car elle met sur un pied d'égalité ceux qui adhérent à l'objectif général de l'organisation. Les moyens de paiement (créditos) sont remis aux participants sous forme de monnaie manuelle avant leur première participation au trueque, et dépendent du type de produit qu'ils proposent : ceux qui offrent des biens produits par eux reçoivent entre 8 et 5 créditos (selon les ferias), contre 2,5 à 4 créditos pour ceux qui revendent des biens qu'ils n’ont pas produits. Cette règle a été instaurée afin d’inciter les 
In Rosario, the reverse is true: getting payment means entails as a prerequisite the divestment of part of one's goods or pesos, as it is possible to obtain 8000 créditos in exchange for 10 pesos.

\author{
The issue, according \\ to Jean-Michel Servet \\ (2010), is to challenge \\ the market principle \\ domination, so as \\ to replace it by \\ reciprocity calling up \\ several solidary \\ alternatives.
}

participants au trueque à ne plus dépendre des politiques d'assistance aux pauvres, afin d'être maîtres de leur propre vie. Mais surtout, on remarque que l'accès aux moyens de paiement ne dépend pas de caractéristiques individuelles, mais de la situation de chacun vis-à-vis du groupe. En cela, le régime de monnayage tend à rappeler que le groupe, en tant que totalité, est toujours présent. D'autre part, ce mode de régulation de l'accès aux moyens de paiement sous-tend une représentation des relations entre les parties et le tout dans laquelle le tout prime sur les parties. En effet, le groupe en tant que tel possède une créance sur ses membres. Dans ce cas, les créditos sont octroyés (sous forme de monnaie manuelle) aux nouveaux membres avant qu'ils ne participent à leur première feria. De plus, la valeur en créditos des moyens de paiement qui leur sont octroyés ne dépend pas de la quantité de biens qu'ils proposent au trueque, mais de leur qualité. C’est donc l'autorité qui, par un principe hiérarchique, permet de participer au trueque ; les membres du nodo sont donc endettés envers elle. À Rosario c'est la situation inverse qui prévaut : afin d'obtenir les moyens de paiement (biens mobilisés dans le règlement des dettes et monnaie manuelle), il est nécessaire de se dessaisir d'une partie de ses biens ou de ses pesos (il est possible d'obtenir 8.000 créditos en échagne de 10 pesos). Or, si le groupe se trouve endetté vis-à-vis de ses membres, celui-ci sera fortement enclin à être instrumentalisé par ceux-là. Il sera alors plus difficile au groupe de se constituer au-delà des divers intérêts privés que si ses membres se trouvent endettés envers lui. Dans ce cas, ceux-ci seront plus à même de s'inscrire en son sein en acceptant les principes qui fondent sa cohésion (et en participant à leur élaboration).

\section{Conclusion}

Le travail effectué sur le terrain par Poriajhú donne un sens concret à la reconnaissance mutuelle des interdépendances s'inscrivant dans une totalité sociale pensée comme telle et, par là, au principe de réciprocité. Il va donc au-delà du constat d'échec du néolibéralisme (en tant qu'idéologie ayant promu l'hégémonie du principe de marché) et incarne l'une des voies possibles du " grand renversement » des principes d'intégration de l'économie à même de constituer une sortie " par le haut » à la crise que nous connaissons actuellement : il s'agit, selon Jean-Michel Servet (2010), de renverser la domination du principe de marché au profit de la réciprocité à travers l'articulation entre une multitude d'alternatives solidaires. C'est en cela, pour reprendre les termes de l'auteur que nous venons de citer, que des expériences " quantitativement insignifiantes » peuvent devenir « qualitativement essentielles ». 
La mise en perspective des expériences de Rosario et Poriajhú met également l'accent sur la dimension monétaire et institutionnelle du grand renversement : puisque la monnaie est à l'image des sociétés, reflétant leurs conflits internes, mais également les valeurs y faisant autorité, le dépassement du néolibéralisme ira de pair avec un bouleversement des formes prises par la monnaie (sur la dimension monétaire de la crise actuelle, voir Théret, 2008a). En effet, la monnaie n’est pas neutre : macroéconomiquement, car elle participe à la détermination du volume de production et de la répartition des revenus, mais aussi socialement, en ce que les modalités d'accès aux moyens de paiement sont à la base des hiérarchies sociales. Ainsi, et au-delà de cet aspect monétaire, pour que le grand renversement soit effectif, il est nécessaire que le renouveau idéologique s’inscrive dans les formes institutionnelles qui étayent l'économie (au sens que la théorie de la Régulation donne à ce terme).

Une question majeure reste cependant en suspend : comment penser un régime de monnayage à même de soutenir l'activité économique tout en mettant ses utilisateurs sur un pied d'égalité ? Sur ce point, le trueque tel qu'il est pratiqué à Poriajhú n'est pas entièrement satisfaisant : il occupe une place relativement marginale dans les budgets familiaux. Ses participants doivent donc impérativement faire usage du peso. Or, du fait de la place marginale occupée par le salariat suite à la désindustrialisation du cordon industriel de Rosario sous la pression des politiques d'inspiration néolibérales, ils sont doublement dominés à travers le régime de monnayage des monnaies de crédit bancaire : ils dépendent des dépenses effectuées par les salariés, qui elles-mêmes dépendent de celles effectuées par les propriétaires des moyens de production. En effet, seuls ces derniers ont accès au crédit leur permettant de mettre en œuvre leur production. Une partie de leurs dépenses prend la forme des salaires (Cartelier 2007 ; Orléan 2008), qui doivent ensuite être dépensés par les salariés afin de former le revenu de ceux qui ne sont ni salariés ni capitalistes. La question du régime de monnayage se pose avec d'autant plus d'acuité qu'une telle configuration n'est pas propre à Capitán Bermúdez : il est possible de considérer que le salariat occupe une place marginale dans de nombreux pays du Sud dans lesquels prévalent ce que Keith Hart (1973) qualifie d' « activités économiques informelles ». Dans la plupart des cas, cette double domination se traduit par une grande fluctuation des revenus, qui trouve son origine dans l'inégal accès aux moyens de paiements.
As currency mirrors societies, reflecting their internal conflicts and values, overcoming neoliberalism will go together with a thorough change in currency forms (on the monetary dimension of the present crisis, see Theret, 2008a)

One major issue stays pending: how is it possible to conceive of a currency system able to sustain economic activity while putting all users on an equality footing? 


\section{Bibliographie}

- Aglietta, Michel. 1988. L'ambivalence de l'argent. Revue française d'économie 3 (3):87-133.

- _ 2007. Universalité et transformations de la monnaie : la nature des crises monétaires. In $L a$ monnaie dévoilée par ses crises, edited by B. Théret. Paris: Editions de l’École des Hautes Études en Sciences Sociales.

- Aglietta, Michel, Jean Andreau, Marc Anspach, Jacques Birouste, Jean Cartelier, Daniel De Coppet, Charles Malamoud, André Orléan, Jean-Michel Servet, Bruno Théret, and Jean-Marie Thivaud. 1998. Introduction. In La monnaie souveraine, edited by M. Aglietta and A. Orléan. Paris: Odile Jacob.

- Aglietta, Michel, and Jean Cartelier. 1998. Ordre monétaire des économies de marché. In La monnaie souveraine, edited by M. Aglietta and A. Orléan. Paris: Odile Jacob.

- Aglietta, Michel, and André Orléan. 2002. La monnaie entre violence et confiance. Paris: Odile Jacob.

• — - eds. 1998. La monnaie souveraine. Paris: Odile Jacob.

- Alary, Pierre. 2006. Les dynamiques monétaires et le développement des échanges marchands, Economie, EHESS, Paris.

- Blanc, Jérôme. 1997. Monnaie, confiance et temps. In La construction sociale de la confiance, edited by J.-M. Servet and P. Bernoux. Paris: Montchrestien.

- 2000. Les monnaies parrallèles. Paris: L’Harmattan.

• — nomica.

- Cartelier, Jean. 2007. The hypostasis of money: an economic point of view. Cambridge Journal of Economics (37):217-233.

- Coppet, Daniel de. 1998. Une monnaie pour une communauté mélanésienne comparée à la nôtre pour l’individu des sociétés européennes In La monnaie souveraine, edited by M. Aglietta and A. Orléan. Paris: Odile Jacob.

- Courbis, Bernard, Eric Froment, and Jean-Michel Servet. 1990. A propos du concept de monnaie. Cahiers d'économie politique 18:5-29.

- Dumont, Louis. 1983. Essais sur l'individualisme une perspective anthropologique sur l'idéologie moderne, Esprit. Paris: Le Seuil.

- Gómez, Georgina. 2008. Making markets. The institutional rise and decline of the Argentine Red de Trueque, Development Studies, Institute of Social Studies, La Haye.

- Guérin, Isabelle. 2008. L’argent des femmes pauvres : entre survie quotidienne, obligations familiales et normes sociales. Revue française de socio-économie (2):59-78.

- Hart, Keith. 1973. Informal Income Opportunities and Urban Employment in Ghana The Journal of Modern African Studies 11 (1):61-69.

- Hillenkamp, Isabelle. À paraître: $c f$. ce numéro.

- Ingham, Geoffrey K. 2004. The nature of money. Cambridge: Polity.

- Luzzi, Mariana. 2005. Réinventer le marché ? Les clubs de troc face à la crise en Argentine. Edited by J. Blanc. Paris: L’Harmattan.

- Nigris, A de. 2008. La bancarización en Argentina In Serie Financiamiento del desarrollo $n^{\circ} 204:$ CEPAL.

- Orléan, André. 2002. La monnaie contre la marchandise. L'homme, Revue Française d'Anthropologie (162):27-48.

- _ 2008. Monnaie, séparation marchande et rapport salarial. In Conflits et pouvoirs dans les institutions du capitalisme, edited by F. Lordon. Paris: Presses de la Fondation des Sciences Politiques.

- Parry, Jonathan P., and Maurice Bloch, eds. 1989. Money and the morality of exchange. Cambridge [England] ; New York: Cambridge University Press.

- Plasencia, Maria Adela, and Ricardo Orzi, eds. 2007. Moneda Social y Mercados Solidarios. BuenosAires: Ciccus. 
- Polanyi, Karl. 1983 [1944]. La grande transformation : aux origines politiques et économiques de notre temps. Paris: Gallimard.

- Polanyi, Karl, Conrad Maynadier Arensberg, and Harry W. Pearson, eds. 1957. Trade and market in the early empires : economies in history and theory. Glencoe, Ill: The Free Press.

· Servet, Jean-Michel. 2007. Le principe de réciprocité chez Karl Polanyi. Revue tiers monde 190:255273.

- 2010. Le grand renversement. De la crise au renouveau solidaire. Edited by J.-L. Laville, Solidarité et société. Paris: Desclée de Brouwer.

• —_ ed. 1999. Une économie sans argent : les systèmes d'échange local. Paris: Seuil.

- Théret, Bruno. 1998. La dualité des dettes et de la monnaie dans les sociétés salariales. In La monnaie souveraine, edited by M. Aglietta and A. Orléan. Paris: Odile Jacob.

. 2008a. L’argent de la mondialisation: en quoi pose-t-il des problèmes éthiques? Un point de vue régulationnniste commonsien. Sciences Politiques Comparées (10):1-40.

- _ 2008b. Les trois états de la monnaie. Approche interdisciplinaire du fait monétaire. Revue économique 59 (4):813-841.

• — 2009. Monnaie et dettes de vie. A propos de quelques critiques adressées à La monnaie souveraine. L'Homme, Revue française d'anthropologie (190):153-180.

• —_ ed. 2007. La monnaie dévoilée par ses crises. 2 vols, Civilisations et sociétés,. Paris: Éditions de l'École des hautes études en sciences sociales.

- Théret, Bruno, and Miguel Zanabria. 2009. Sur la pluralité des monnaies publiques dans les fédérations. Une approche de ses conditions de viabilité à partir de l'expérience argentine récente. Economie et institutions:40. 


\section{ANNEXES}

Annexe 1 : comparaison des évaluations monétaires (El campito)

\begin{tabular}{|lrrr|}
\hline Produit & Créditos & Pesos & Créditos/peso \\
\hline Hors aide et légumes & & & \\
Farine (1 Kg) & 3500 & 1,30 & 2692 \\
Sucre (1 Kg) & 3500 & 2,20 & 1591 \\
Sel (1Kg) & 2000 & 2,20 & 909 \\
Savon (1) & 3500 & 1,50 & 2333 \\
Pure de tomate (1) & 3500 & 2,00 & 1750 \\
Pâtes (500g) & 3500 & 1,50 & 2333 \\
Moyenne & & & 1935 \\
\hline Aide aux pauvres & & & \\
Lait en poudre & 8000 & $>10$ & $<800$ \\
\hline Légumes & & & \\
Tomate & 2500 & 4,00 & 1818 \\
Oignon & 2000 & 1,10 & 1333 \\
Carottes & 1000 & 0,75 & $400-500$ \\
Calabaza & $2000-2500$ & 5,00 & 1000 \\
Zapallito (1) & 1000 & 1,00 & 955 \\
Moyenne & & & \\
\hline Vêtements & & & \\
Divers & $>1000$ & & \\
\hline
\end{tabular}

Source: enquête de terrain.

Annexe 2 : moyens de paiement (monnaie manuelle), Poriajhú
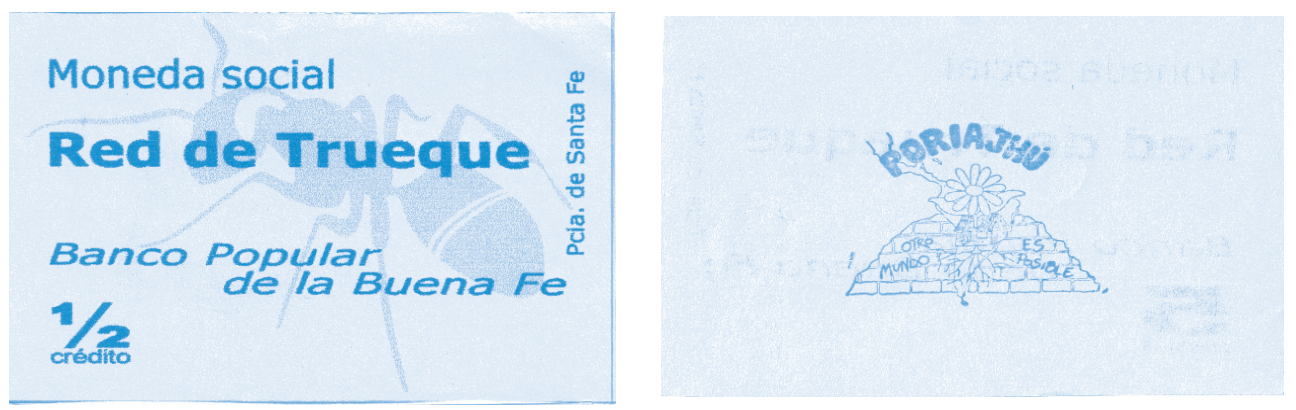